EUROPEAN ORGANIZATION FOR NUCLEAR RESEARCH

CERN-PPE/92-206

24 November 1992

\title{
Performance of a Liquid Argon Preshower Detector Integrated with an Accordion Calorimeter
}

\section{RD3 Collaboration}

B.Aubert, A.Bazan, B.Beaugiraud, J.Colas, T.Leflour, M.Maire,

J.P.Vialle, I.Wingerter-Seez and Y.P.Zolnierowski LAPP, Annecy, France

H.A.Gordon, V.Radeka, D.Rahm, and D.Stephani Brookhaven National Laboratory, Upton, USA

N.Bulgakov, J.L.Chevalley, C.W.Fabjan, D.Fournier ${ }^{\dagger}$ O.Gildemeister, P.Jenni, M.Nessi, F.Nessi-Tedaldi, M.Pepe, W.Richter, J.Soderqvist and V.Vuillemin

CERN, Geneva, Switzerland

J.M.Baze, L.Gosset, P.Lavocat, J.P.Lottin, B.Mansoulie, J.P.Meyer, J.F.Renardy, J.Teiger, and H.Zaccone DAPNIA-SPP Saclay, Gif-sur-Yvette, France

G.Battistoni, D.V.Camin, D.Cavalli, G.Costa, A.Cravero, A.Ferrari, F.Gianotti, L.Mandelli, M.Mazzanti, L.Perini, G.Pessina and M.Sciamanna

Dipartimento di Fisica dell'Università e Sezione INFN, Milano, Italy

E.Augé, R.Chase, J.C.Chollet, C.de La 'Taille, L.Fayard, A.Hrisoho, Ph.Jean, G.Le Meur, B.Merkel, J.M.Noppe, G.Parrour, P.Pétroff, J.P.Repellin, A.Schaffer, N.Seguin and G.Unal LAL, Orsay, France

C.Fuglesang

Manne Siegbahn Institute, Stockholm, Sweden

M.Lefebvre

University of Victoria, Canada

submitted to Nucl. Instr. Meth. 


\begin{abstract}
A prototype liquid argon preshower detector with a strip granularity of $2.5 \mathrm{~mm}$ has been tested at the CERN SPS in front of a liquid argon Accordion calorimeter. For charged tracks a signal-to-noise ratio of 9.4 and a space resolution of $340 \mu \mathrm{m}$ were measured; the rejection power against overlapping photons produced in the decay of $50 \mathrm{GeV} \pi^{0}$ 's is larger than 3 ; the precision on the electromagnetic shower direction, determined together with the calorimeter, is better than $7 \mathrm{mrad}$ above $40 \mathrm{GeV}$; the calorimeter performance behind the preshower $\left(\sim 4 \mathrm{X}_{0}\right)$ is fully preserved. These results make such a detector attractive for future operation at the CERN Large Hadron Collider.
\end{abstract}

\footnotetext{
"Visitor from IHEP, Protvino, Russia

'also LAL, Orsay, France
} 


\section{Introduction}

The use of a finely segmented preshower detector for complementing the calorimeter and tracking information in experiments at the future hadron colliders is motivated by several arguments. Such a device can distinguish single $\gamma$ 's from a background of overlapping photons mainly produced in the decay of energetic $\pi^{0}$ 's. A preshower can contribute to the electron identification by providing additional rejection against charged hadrons, either isolated or with overlapping photons. This detector is also useful for recovering the energy lost by electrons and photons in the material upstream of the calorimeter and therefore for preserving the calorimeter performance. Furthermore a combined measurement of the shower positions in the calorimeter and in the preshower provides a precise determination of the particle direction.

In case of a liquid argon (LAr) calorimeter the best location for a presampler is inside the cryostat just in front of the calorimeter: this configuration has the advantage of minimizing the deterioration of the calorimeter energy resolution due to the early shower development in the cryostat and in the preshower material while allowing for a compact integrated system.

Over the last two years we have been developping the project of fast LAr calorimetry $[1,2]$ for experimentation at the CERN Large Hadron Collider (LHC), based on a new geometry in which the absorber and readout plates are bent with an "accordion" shape and connected to fast preamplifiers mounted on the detector faces. This design minimizes the charge transfer time from the calorimeter to the readout chain and thus allows to exploit the rapid rise of the ionization current produced by a shower in the liquid. At the same time it provides a hermetic detector with no dead space in between towers. The feasibility of this concept has been demonstrated by extensive beam tests of two electromagnetic prototypes with parallel and cylindrical geometry $[3,4,5]$.

In the meantime we have explored the potential of an integrated LAr preshowercalorimeter system by testing a small size presampler in front of one of the Accordion prototypes at the CERN SPS. The main results are reported in this paper: the detector geometry and readout are described in section 2 , and the experimental setup for data taking in section 3; the performances obtained with muons, electrons and photons are discussed in section 4 to 6 , while section 7 is devoted to the conclusions.

\section{Detector Geometry and Readout}

The preshower prototype has a transverse size of $6 \mathrm{~cm} \times 6 \mathrm{~cm}$ and consists (Fig. 1) of two $7 \mathrm{~mm}$ thick sheets of $\mathrm{Pb}$ interleaved with two $10 \mathrm{~mm}$ thick active layers of LAr, resulting in a sampling fraction of about $15 \%$ (similar to that of the Accordion calorimeter).

Each LAr layer is equipped with 24 signal electrodes ( $\mathrm{Cu}$-Kapton strips), $6 \mathrm{~cm}$ long and $300 \mu \mathrm{m}$ thick, alternated with ground electrodes. In the two layers the strips are arranged along two orthogonal directions to determine the particle position in the vertical ( $y$, first layer) and in the horizontal ( $x$, second layer) views. The LAr gap on either side of each electrode is $0.9 \mathrm{~mm}$ and the total electron drift time $\sim 200 \mathrm{~ns}$. The resulting cell capacitance is about $16 \mathrm{pF}$.

The strips are tilted by an angle of $14^{\circ}$ so that a straight track perpendicular to the prototype surface crosses two adjacent cells in each layer: this ensures full geometrical efficiency and improves the space resolution of the detector. The effective granularity is $2.54 \mathrm{~mm} \times 60 \mathrm{~mm}$.

For the beam tests the operating voltage across the gap was set to $1 \mathrm{kV}$. 
The frontend electronics consisted of low noise, high sensitivity charge preamplifiers (PA) based on GaAs MESFETs, similar to those used for the calorimeter readout [6]. They have an input capacitance of $2.5 \mathrm{pF}$, a feedback capacitance of $1 \mathrm{pF}$ and an input impedance of $120 \Omega$; the power dissipation is about $50 \mathrm{~mW}$. To minimize the charge transfer time they were directly mounted on the detector.

Bipolar shapers with a response peaking time of $30 \mathrm{~ns}$ to a fast $(\delta)$ pulse and $55 \mathrm{~ns}$ to the triangular current signal coming from the preshower were used in the second stage amplification. These were located outside the cryostat and were followed by 12 -bit charge integrating ADC LeCroy 2282 operated with a gate width of $15 \mathrm{~ns}$ (for this purpose the LeCroy system processor was suitably modified).

With $55 \mathrm{~ns}$ peaking time the average electronic noise, determined from the fluctuations of the ADC pedestals, was 1.8 counts per channel (Equivalent Noise Charge about 1200 rms electrons).

A channel-to-channel equalization to within $\pm 10 \%$ was performed offline by using muon scan data, while the overall energy scale, about 1.4 (incident) $\mathrm{MeV} /$ count, was determined with an electron beam (see section 5.2).

\section{Test Beam Setup}

For the beam tests the preshower was installed in front of the Accordion prototype with cylindrical geometry, whose performance is described in [5]. The calorimeter has a granularity of $2.7 \mathrm{~cm} \times 2.5 \mathrm{~cm}$ in the $x \times y$ direction and in the region covered by the preshower is segmented in two longitudinal compartments of thickness $13.4 \mathrm{X}_{0}$ and $12 \mathrm{X}_{0}$ respectively. The calorimeter was read out with a fast electronic chain providing bipolar shaped signals with peaking time $35 \mathrm{~ns}$.

Preshower and calorimeter were cooled down inside the same cryostat: the vessel consisted of a $3 \mathrm{~mm}$ thick stainless steel outer wall followed by $3 \mathrm{~cm}$ of vacuum and by a $2 \mathrm{~cm}$ thick aluminium wall. There was about $15 \mathrm{~cm}$ between the inner vessel and the preshower, mostly filled with low density "Rohacell" foam, and $5 \mathrm{~cm}$ of LAr between the preshower and the Accordion front face. The resulting amount of material before the first (second) active layer was $2.1 \mathrm{X}_{0}\left(3.4 \mathrm{X}_{0}\right)$, contributing to a total of about $3.8 \mathrm{X}_{0}$ in front of the calorimeter.

The cryostat was mounted on a platform which could be moved in the horizontal and vertical direction and rotated by up to $\pm 4^{\circ}$ about its vertical axis.

The tests were performed in the $\mathrm{H} 6$ beam line of the CERN SPS with electrons and photons; muon data were also collected by exploiting the large $\mu$ contamination in the electron beam above $150 \mathrm{GeV} / \mathrm{c}$.

The beam line was equipped with two fast scintillation counters, used in the trigger, and three proportional wire chambers $(B C)$ which allowed to reconstruct the particle impact point at the front face of the preshower with a precision of about $290 \mu \mathrm{m}(470 \mu \mathrm{m})$ in the horizontal (vertical) direction. The information from the scintillators and the BC was also used in the offline analysis to select clean muon and electron events by requiring signals compatible with a single charged track.

\section{Response to Muons}

The preshower capability of detecting minimum ionizing particles was studied with muon events extracted from high energy electron runs by requiring a small energy deposition in the calorimeter. 
Due to the tilted strips, the muon signal was reconstructed in each layer by adding the contributions of two adjacent cells: the most energetic one and its neighbour with more energy. The pedestals of the electronic chain were determined from events taken with a random trigger during the same bursts as the muons. An example of a reconstructed $\mu$ spectrum is shown in Fig. 2: the most probable energy loss ("1 mip" peak) corresponds to about $16 \mathrm{ADC}$ counts per layer.

The electronic noise was measured in the random trigger events mentioned above by looking at the energy contained in four channels (two per layer). The resulting distribution, also plotted in Fig. 2, is well separated from the muon spectrum and a fit with a Gaussian shape gives $\sigma_{\text {noise }} \simeq 3.5$ counts $(\sim 1.8$ counts/channel). The signal-to-noise ratio is therefore $9.4 \pm 0.9$ over the total preshower depth and $6.7 \pm 0.8$ in the single layers. Due to this good performance the reconstructed muon signal is not biased to higher energy, as demonstrated by the fact that the same spectrum and peak position were obtained by summing the energy contained in two cells per layer around the particle impact point provided by the beam chamber system.

The energy sharing between adjacent channels allows to reconstruct the muon position, in each view, as the energy weighted barycentre of a two cell cluster. Therefore a somewhat better performance is expected than in a detector with the same granularity but with straight electrodes.

The space resolution of the prototype was determined by fitting with a Gaussian shape the distribution of the difference between the muon position measured by the preshower and the particle impact point extrapolated from the beam chamber hits (Fig. 3): after unfolding the $\mathrm{BC}$ contribution, the resolution is $340 \pm 40 \mu \mathrm{m}$ in both views.

\section{Response to Electrons}

The results discussed in this section are based on electron data collected with various beam energies between 10 and $197 \mathrm{GeV} / \mathrm{c}$. The particles entered the two prototypes at normal incidence and were distributed over a spot size of about $1 \mathrm{~cm}^{2}$.

\subsection{Electron signal in the preshower}

An example of the electromagnetic shower profiles in the preshower is shown in Fig. 4: more than $70 \%$ of the energy is contained in three neighbouring cells. The rms shower size is typically $2 \mathrm{~mm}(2.5 \mathrm{~mm})$ in the first (second) layer, weakly decreasing with energy.

The average signal in the active preshower part due to electrons of different energies is shown in Fig. 5. In the first layer the signal varies between 20 and 70 mip over the investigated range, while in the second layer, which is preceeded by one more radiation length, it is two to four times higher. The probability for a $10 \mathrm{GeV}$ electron to give more than $3 \mathrm{mip}$ in the first (second) layer is $98 \%(100 \%)$ : this indicates the potential rejection capability of a cut on the minimum preshower signal to distinguish electrons from charged hadrons (see Fig. 2 and section 4 ).

An important requirement for a preshower operating at the future hadron colliders will be an electronic dynamic range large enough to detect minimum ionizing tracks as well as to measure the large signals from high energy electrons produced in physics channels of prime interest, such as a possible heavy $Z^{\prime} \rightarrow e^{+} e^{-}$production. An indication of this range can be obtained by extrapolating a parametrization of the test beam data to the $\mathrm{TeV}$ region. Figure 6 shows the signal value in the most energetic channel of a given 
preshower layer corresponding to $95 \%$ electron efficiency. It can be seen for instance that $95 \%$ of $150 \mathrm{GeV}$ electrons will produce less than $150 \mathrm{mip}$ in the highest energy cell of the second layer. An extrapolation of the observed trend indicates that even with a relatively early shower sampling (after about $3 \mathrm{X}_{0}$ ) a dynamic range of 5000 would be needed to measure with a linear response signals from up to $1 \mathrm{TeV}$ electrons while maintaining the capability of detecting the " 1 mip" peak at 9-10 standard deviations above the noise. Behind $2 \mathrm{X}_{0}$ (first layer) the expected dynamic range is a factor of five smaller.

\subsection{Energy measurement with the preshower-calorimeter system}

By using the information of the calorimeter and preshower detector the incident electron energy $\left(E_{t o t}\right)$ was reconstructed as

$$
E_{\text {tot }}=E_{\text {calo }}+\alpha Q_{P S}
$$

where $E_{\text {calo }}$ is the energy measured in the calorimeter, $Q_{P S}$ the total signal (in ADC counts) from the active preshower part and $\alpha$ a suitable weight factor.

In the calorimeter the electromagnetic shower was reconstructed, in each of the two compartments, by summing the energy deposited in a matrix of $3 \times 3$ cells $\left(\sim 8 \times 8 \mathrm{~cm}^{2}\right)$ around the channel with the largest signal ${ }^{1}$. The variation of the Accordion response with the particle impact point over the cell was corrected as described in $[4,5]$.

The weight $\alpha$, which depends on the total amount of material upstream of the calorimeter, was determined by minimizing the width of the $E_{\text {tot }}$ distribution and was found to be around $1.4 \mathrm{MeV} /$ count, slightly increasing for electron energies below $50 \mathrm{GeV}$.

The average energy fraction $\left\langle\alpha Q_{P S} / E_{\text {tot }}\right\rangle$ released by electrons in the $3.8 \mathrm{X}_{0}$ preceding the calorimeter is plotted in Fig. 7 as a function of the beam momentum: it decreases rapidly from $23 \%$ at $10 \mathrm{GeV}$ to $3.5 \%$ at $200 \mathrm{GeV}$ and, by extrapolation, to $1.4 \%$ at $1 \mathrm{TeV}$.

Also shown in Fig. 7 are the rms values: they scale approximately as $24 \% / \sqrt{E(G e V)}$, which is a factor of two larger than the intrinsic Accordion resolution. The calorimeter measurement would therefore be seriously spoiled if the energy lost in the upstream material were not corrected for. As an example Fig. 8 compares the spectra obtained for $119 \mathrm{GeV}$ electrons when only the calorimeter or both the calorimeter and the preshower information is used: in the latter case the $1 \%$ Accordion resolution is restored.

\subsubsection{Energy resolution}

The energy resolution of the preshower-calorimeter system is plotted in Fig. 9 as a function of the incident electron energy (beam momentum spread unfolded). The experimental points can be fitted as

$$
\frac{\sigma(E)}{E}=\frac{10.0 \pm 0.5}{\sqrt{E}} \% \oplus(0.5 \pm 0.1) \% \oplus \frac{0.343 \pm 0.028}{E}
$$

where $E$ is in $\mathrm{GeV}$ and the last term is the contribution of the electronic noise of the calorimeter $(\sim 72 \mathrm{MeV} /$ cell). A comparison with the result obtained in the Accordion region not covered by the preshower (Fig.9 and [5])

$$
\frac{\sigma(E)}{E}=\frac{10.0 \pm 0.5}{\sqrt{E}} \% \oplus(0.6 \pm 0.1) \% \oplus \frac{0.319 \pm 0.041}{E}
$$

demonstrates that the calorimeter response is fully preserved.

\footnotetext{
${ }^{1}$ This nonet of towers contains about $92 \%$ of the shower energy.
} 
The performance of an alternative preshower structure in which the passive material is concentrated in one block in front of the active part was simulated by using only the signal from the second layer for the energy measurement. The resolution obtained in this way, also shown in Fig. 9, is the same as in the previous cases above $50 \mathrm{GeV}$ but gradually deteriorates below. This results in an increase of the sampling term to $11.1 \% / \sqrt{E}$. Note however that in this configuration only $1 \mathrm{~cm}$ of instrumented LAr was read out.

Finally, the result (2) is only marginally improved if the factor $\alpha$ in (1) is replaced by two different weights for the signals coming from the two layers, which could be justified by the larger amount of passive material in front of the first layer than in between the two layers. On the other hand the resolution is not deteriorated if a smaller preshower area, three cells per view, is used to reconstruct the shower energy.

\subsubsection{Linearity}

Fig. 10 shows, for each momentum setting, the mean electron energy deposition in the combined preshower-calorimeter, taken as the peak of the Gaussian fit to the $E_{\text {tot }}$ distribution, normalized to the nominal beam energy. The energy scale has been determined by the $119 \mathrm{GeV}$ point. The errors include an uncertainty of $\pm 100 \mathrm{MeV}$ from the magnet hysteresis.

The response linearity of the combined preshower-calorimeter system is well within $\pm 1 \%$ both when two preshower layers or only the second one are used for the energy measurement.

\subsection{Space resolution}

The electromagnetic shower position was reconstructed in each view as the energy weighted barycentre of three adjacent cells consisting of the most energetic one and its two neighbours (Fig. 4). Test beam data and Monte Carlo simulations of the preshower geometry show that the best space resolution is achieved when this minimal cluster size is used. This was attributed to the fact that a three cell region includes only the shower core and is therefore less subject to the fluctuations in the broad tails.

After correcting the reconstructed electron position for a bias toward the cell centre, usual in pad detectors, the resolution shown in Fig. 11 as a function of the beam energy was obtained. Before unfolding the beam chamber contribution the experimental points can be fitted as

$$
\begin{aligned}
& \sigma\left(x_{P S}-x_{B C}\right)=\frac{1.91 \pm 0.09}{\sqrt{E}} \oplus 0.30 \pm 0.01 \mathrm{~mm} \\
& \sigma\left(y_{P S}-y_{B C}\right)=\frac{2.37 \pm 0.07}{\sqrt{E}} \oplus 0.47 \pm 0.01 \mathrm{~mm}
\end{aligned}
$$

where $E$ is in $\mathrm{GeV}$. The constant terms are compatible with the resolution of the $\mathrm{BC}$ system in the two views quoted in section 3 , while the lower sampling term in $x$ is due to the larger energy deposition in the second layer. The contribution of the electronic noise, which is significant for muons, is negligible for electromagnetic showers, amounting to about $20 \mu \mathrm{m}$ at $10 \mathrm{GeV}$ (i.e. in the worst case). The results (4) and (5) show that above $90 \mathrm{GeV}$ the preshower space resolution, after unfolding the beam chamber contribution, is in the range $150-250 \mu \mathrm{m}$. 


\subsection{Angular resolution of the preshower-calorimeter system}

The preshower and calorimeter information can be combined to determine the direction of the incoming electron through the measurements of the shower position at two consecutive depths. The angle of the incident particle is then given, for instance in the $x$ direction, by

$$
\vartheta_{x}=\arctan \left(\frac{x_{C A L O}-x_{P S}}{d_{x}}\right)
$$

where the numerator is the difference between the shower $x$ positions reconstructed in the two detectors and the denominator is their average longitudinal separation. The latter can be evaluated by means of angular scan data, which were only taken with $80 \mathrm{GeV}$ incident photons, and was found to be (see section 6.1) $d_{x}=140 \pm 3 \mathrm{~mm}\left(d_{y}=173 \pm 3\right.$ $\mathrm{mm})$. For electrons, taking into account the different longitudinal profile of electron and photon-induced showers [7], it was assumed $d_{x}=125 \mathrm{~mm}\left(d_{y}=158 \mathrm{~mm}\right)$.

In the preshower the electron position was reconstructed as described in section 5.3. In the calorimeter the $x(y)$ position was determined as the energy weighted barycentre of a cluster of $5 \times 3(3 \times 3)$ cells in the $x \times y$ plane. The choice of a larger cluster for the $x$ measurement was dictated by the position of the beam spot, which covered the boundary between two adjacent cells in $x$, and allowed to reduce the bias due to edge effects. Only the first compartment of the Accordion $\left(13.4 \mathrm{X}_{0}\right)$, which contains more than $80 \%$ of the total shower energy, was used for this measurement: adding the information from the second sampling would spoil the resolution because the energy deposition is small and therefore the reconstructed barycentre is affected by the electronic noise ${ }^{2}$.

The direction measured according to (6) for an incident $60 \mathrm{GeV}$ electron beam is plotted in Fig. 12: the Gaussian fit to this distribution gives an angular accuracy of about 3.5 mrad. The resolution obtained for all energy points is shown in Fig. 13 and can be fitted as

$$
\begin{aligned}
\sigma_{\theta}^{x} & =\frac{32.2 \pm 3.7}{\sqrt{E}} \oplus 4.1 \pm 0.2 \oplus \frac{132.2 \pm 21.0}{E} \operatorname{mrad} \\
\sigma_{\theta}^{y} & =\frac{18.8 \pm 2.1}{\sqrt{E}} \oplus 2.3 \pm 0.1 \oplus \frac{63.7 \pm 10.3}{E} \mathrm{mrad}
\end{aligned}
$$

where $E$ is in $\mathrm{GeV}$. The contribution of the beam divergence was estimated to be less than $0.2 \mathrm{mrad}$. The above result is dominated by the performance of the calorimeter, whose space resolution [5] is typically a factor of two to three worse than that of the preshower. The last terms in (7) and (8) are compatible with the contribution expected from the calorimeter electronic noise, which is higher in $x$ due to the larger number of cells included in the cluster. The better resolution in $y$ can also be attributed to the longer lever arm $d_{y}$ and to the positioning of the beam spot on an $x$ cell boundary in the calorimeter.

Finally, the result in (7) can be compared to the $x$ angular resolution obtained with the calorimeter alone, using the shower barycentres reconstructed in the first two compartments (thickness $\sim 2 \times 8 \mathrm{X}_{0}$ ) in a region segmented in three longitudinal samplings [5]. Above $100 \mathrm{GeV}$ the performance is similar, while at lower energy the precision of the preshower-calorimeter measurement is better by up to $30 \%$.

\footnotetext{
${ }^{2}$ The longitudinal segmentation of the calorimeter in the region covered by the preshower was not optimized for angular measurements.
} 


\section{Response to Photons}

For photon data the test setup described in section 3 was modified as shown in Fig. 14. Photons were produced by inserting a $0.5 \mathrm{~mm}$ thick lead foil into the electron beam path. A bending magnet installed behind the radiator separated electrons from photons in the vertical direction. The electrons were bent to a calorimeter region not covered by the preshower, while the photons traversed a $2 \mathrm{~cm}$ thick Fe preconverter followed by a scintillator tag $\left(\mathrm{S}_{5}\right)$ and impinged on the preshower detector. The trigger required a minimum energy deposition in the calorimeter and a good electron hit in a $3 \mathrm{~cm} \times 3 \mathrm{~cm}$ scintillation counter $\left(S_{3}\right)$.

Photons of various energies were selected either by changing the momentum of the primary electron beam ( $148 / \mathrm{c} \mathrm{GeV}$ and $30 \mathrm{GeV} / \mathrm{c}$ settings were used) or by varying the intensity of the current in the bending magnet while keeping fixed the position of the trigger counter $S_{3}$. The obtained $\gamma$ spectrum was between 1 and $130 \mathrm{GeV}$.

Multiple photon radiation in the lead foil was not negligible, as demonstrated by a Monte Carlo simulation of the test setup: at the exit of the radiator about $30 \%$ of the events contained a second $\gamma$ with energy above $1 \mathrm{GeV}$. However, since the conversion probability in the Fe slab was higher for multiple photons, the single photon sample was enhanced by requiring no signal in $S_{5}$. The estimated residual multiple photon contamination in the final event sample was less than $10 \%$.

Due to the later shower development the photon signal in the preshower is typically $30 \%$ lower than that from electrons of comparable energy (Fig. 5) and the lateral shower size is less than $2 \mathrm{~mm}$.

In the calorimeter the electron and photon impact points were aligned in the $x$ direction and separated by about $10 \mathrm{~cm}$ (four cells) in $y$ due to the effect of the bending magnet and the position of the trigger counter. The energy of each of the two showers was reconstructed in a nonet of towers around the particle impact point as explained in section 5.2. For a given current setting in the bending magnet, neither of the two particles had a monochromatic spectrum, due to the finite size of the trigger counter $S_{3}$, but the sum of their energies, including the preshower signal, corresponded to that of the incident beam.

\subsection{Angular resolution}

An accurate determination of the photon direction will be particularly important in LHC experiments to search for a peak in the distribution of the $\gamma \gamma$ invariant mass due to the decay of a Higgs boson in the mass range $80-140 \mathrm{GeV}$.

The direction of the photon shower was determined with the two prototypes as described in section 5.4 for electrons. Furthermore by rotating the platform supporting the cryostat, an angular scan was taken in the $x$ view with incident photons of about 80 $\mathrm{GeV}$. This allowed for a direct determination of the average lever arm $d_{x}$ in (6) from measurements of the mean difference $x_{c a l o}-x_{P S}$ at different rotation angles $\vartheta_{x}$. The result $d_{x}=140 \pm 3 \mathrm{~mm}$ was used over the whole energy range neglecting its logarithmic energy dependence; in the other direction a lever arm $d_{y}=173 \pm 3 \mathrm{~mm}$, obtained by adding to $d_{x}$ the distance between the two preshower active layers, was assumed.

The measured resolution (Fig. 13) was parametrized as

$$
\begin{aligned}
\sigma_{\theta}^{x} & =\frac{36.3 \pm 6.6}{\sqrt{E}} \oplus 2.0 \pm 1.1 \oplus \frac{130.6 \pm 39.3}{E} \mathrm{mrad} \\
\sigma_{\theta}^{y} & =\frac{24.9 \pm 4.1}{\sqrt{E}} \oplus 2.9 \pm 0.3 \oplus \frac{75.8 \pm 28.1}{E} \mathrm{mrad}
\end{aligned}
$$


where $E$ is in $\mathrm{GeV}$.

The angular resolution in the $y$ direction is about $30 \%$ worse than for electrons, due to the particularly small signal produced by photons in the first preshower layer (see Fig. 5).

The accuracy is better than $7 \mathrm{mrad}$ above $40 \mathrm{GeV}$ in both direction: this is a factor of two better than the limit considered acceptable for the search of the intermediate mass Higgs [8], and is also useful for an efficient rejection of the $\pi^{0} \rightarrow \gamma \gamma$ background, as discussed in the next section.

\subsection{Two shower separation}

The ability of detecting a possible $H \rightarrow \gamma \gamma$ signal at the LHC will depend on the experimental capability to adequately reduce the background of $\pi^{0}$ events surviving the set of calorimetric selections against hadronic jets. To achieve the needed rejection of 3 against isolated $\pi^{0}$ 's [8] it will be necessary to recognize the two photons produced in the decay, which are typically a few millimetres apart from each other. This can be done by a granular position-sensitive preshower detector.

The test beam photon data allowed to determine the $2 \gamma$ separation power of the LAr preshower prototype. They covered with enough statistics the spectrum of photons produced in the decay of $50 \mathrm{GeV} \pi^{0}$ 's, potential background to a Higgs of mass about 100 $\mathrm{GeV}$. The decay of a $50 \mathrm{GeV} \pi^{0}$ was simulated by superimposing two single photons with total energy $50 \mathrm{GeV}$ and the correct kinematics. Assuming the preshower detector located at about $140 \mathrm{~cm}$ from the beam line in a future LHC experiment, the distribution of the distance between the two $\gamma$ 's in the detector would peak at the minimum kinematical separation of $7.5 \mathrm{~mm}$ and $96 \%$ of them would be separated by less than $3 \mathrm{~cm}$. At this distance the energy of the softest photon would be about $1 \mathrm{GeV}$.

Two criteria were used to distinguish these overlapped events from single $50 \mathrm{GeV}$ photons. If both $\gamma$ 's from the $\pi^{0}$ convert in the preshower, the broader size of the resulting shower or the presence of two nearby clusters can be exploited. For this purpose a shape variable $(\rho)$ was defined in each layer as the ratio between the energy contained in the three cell cluster mentioned in section 5.1 (Fig. 4) and the total energy released in the layer. As expected, the distribution of $\rho$ (Fig. 15a) peaks at higher values for the narrow single $\gamma$ showers than for the broader double photons. A cut $\rho>0.6$ gives a rejection of 5 against $2 \gamma$ while keeping $91 \%$ of the single photons ${ }^{3}$.

The calorimeter information can be used in combination with the preshower detector to reject those events in which only one of the two $\gamma$ 's from the $\pi^{0}$ decay converts in the presampler. In this case the shower position in the preshower, which is that of the converted photon, will not in general match the shower barycentre reconstructed in the calorimeter, which is instead due to both photons. This method can only be used in the $R \phi$ direction ( $x$ in the test beam geometry) where the position of the interaction vertex is expected to be known with a precision of a few tens of $\mu \mathrm{m}$. Fig. 15b shows the matching between the preshower and the calorimeter barycentres in the $x$ view for the single and the overlapping photon samples (in this latter case only one of the two photons converted in the preshower). A cut at $2 \mathrm{~mm}$, which retains $94 \%$ of the single $\gamma$ events, rejects more than $40 \%$ of double photons.

After convoluting the efficiency and rejection of these two selections with the conversion probability of $\gamma$ 's from $\pi^{0}$ decay, the result shown in Fig. 16 was obtained: the needed

\footnotetext{
${ }^{3}$ Simulation studies reproducing similar detector granularity and shaping time as in the beam test concluded that the deterioration of the single photon efficiency due to the pile-up of several soft interactions on top of the physically interesting event when operating at a luminosity of $1.7 \times 10^{34} \mathrm{~cm}^{-2} \mathrm{~s}^{-1}$ should be less than $5 \%$.
} 
rejection factor of 3 against isolated $\pi^{0}$ 's can be safely achieved for an acceptable efficiency of $90 \%$ on single photons.

\section{Conclusions}

The performance of a compact LAr system, consisting of a highly segmented preshower detector followed by an Accordion electromagnetic calorimeter at a few centimetre distance, has been explored through the beam tests of small prototypes. Both detectors were read out with electronic chains fast enough to cope with the expected interaction rates at the future CERN Large Hadron Collider.

The signal from charged tracks was detected in the preshower at more than 9 standard deviations from the electronic noise and their position reconstructed with a resolution of $340 \mu \mathrm{m}$.

The high space accuracy obtained with electromagnetic showers $(\sim 2 \mathrm{~mm} / \sqrt{E(\mathrm{GeV})})$ can be used in the electron identification through precise track-preshower matching techniques.

The angular resolution provided by the preshower-calorimeter system is better than 7 mrad above $40 \mathrm{GeV}$ and the rejection factor against $50 \mathrm{GeV} \pi^{0}$ 's is larger than 3 .

The calorimeter performance behind the preshower detector is fully preserved if the signal from the active preshower part is used to measure the energy lost by electrons and photons in the passive material.

A dedicated effort towards the construction of larger size preshower prototypes and the development of low noise, small power consuming and radiation hard readout electronics is presently under way within our R\&D activity for LHC [9].

\section{Acknowledgements}

We are grateful to the technical staffs of the collaborating institutes for their contributions. In particular we would like to thank L.Baisin, J.C.Berset, L.Bonnefoy, G.Dubois-Dauphin, C.Marin, R.Poncet, A.Sigrist.

Financial support is acknowledged from the Institut National de Physique Nucléaire et de Physique des Particules for the Annecy and Orsay groups, from the Istituto Nazionale di Fisica Nucleare for the Milano group and from the US Department of Energy for the BNL group. 


\section{References}

[1] B.Aubert et al. (RD3 Collaboration), "Liquid Argon Calorimetry with LHCPerformance Specifications", CERN/DRDC/90-31 (1990).

[2] B.Aubert et al. (RD3 Collaboration), "Hadronic and Electromagnetic Liquid Argon LHC Prototype Calorimeter with Pointing Geometry", CERN/DRDC/91-21 (1991).

[3] B.Aubert et al. (RD3 Collaboration), "Performance of a Liquid Argon Electromagnetic Calorimeter with an Accordion Geometry", Nucl. Inst. and Meth. A309(1991)438.

[4] B.Aubert et al. (RD3 Collaboration), "Performance of a Liquid Argon Accordion Calorimeter with Fast Readout", CERN-PPE/92-46, to be published in Nucl. Instr. and Meth.

[5] B.Aubert et al. (RD3 Collaboration), "Performance of a Liquid Argon Electromagnetic Calorimeter with a Cylindrical Accordion Geometry", CERNPPE/92-129, to be published in Nucl. Instr. and Meth.

[6] D.V.Camin, G.Pessina and E. Previtali, "Gallium Arsenide Low-Noise Preamplifiers for Cryogenic Particle Detectors", to be published in Proc. of the Erice Workshop on GaAs Detectors and Electronics, Erice, Italy, 12-18 January 1992.

[7] Particle Data Group, Phys. Rev. D11(1992)III.16

[8] ATLAS Collaboration, Letter of Intent for a General-Purpose $p p$ Experiment at the Large Hadron Collider at CERN, CERN/LHCC/92-4.

[9] RD3 Collaboration, R\&D for a Liquid Argon Preshower, CERN/DRDC/92-40 (1992). 


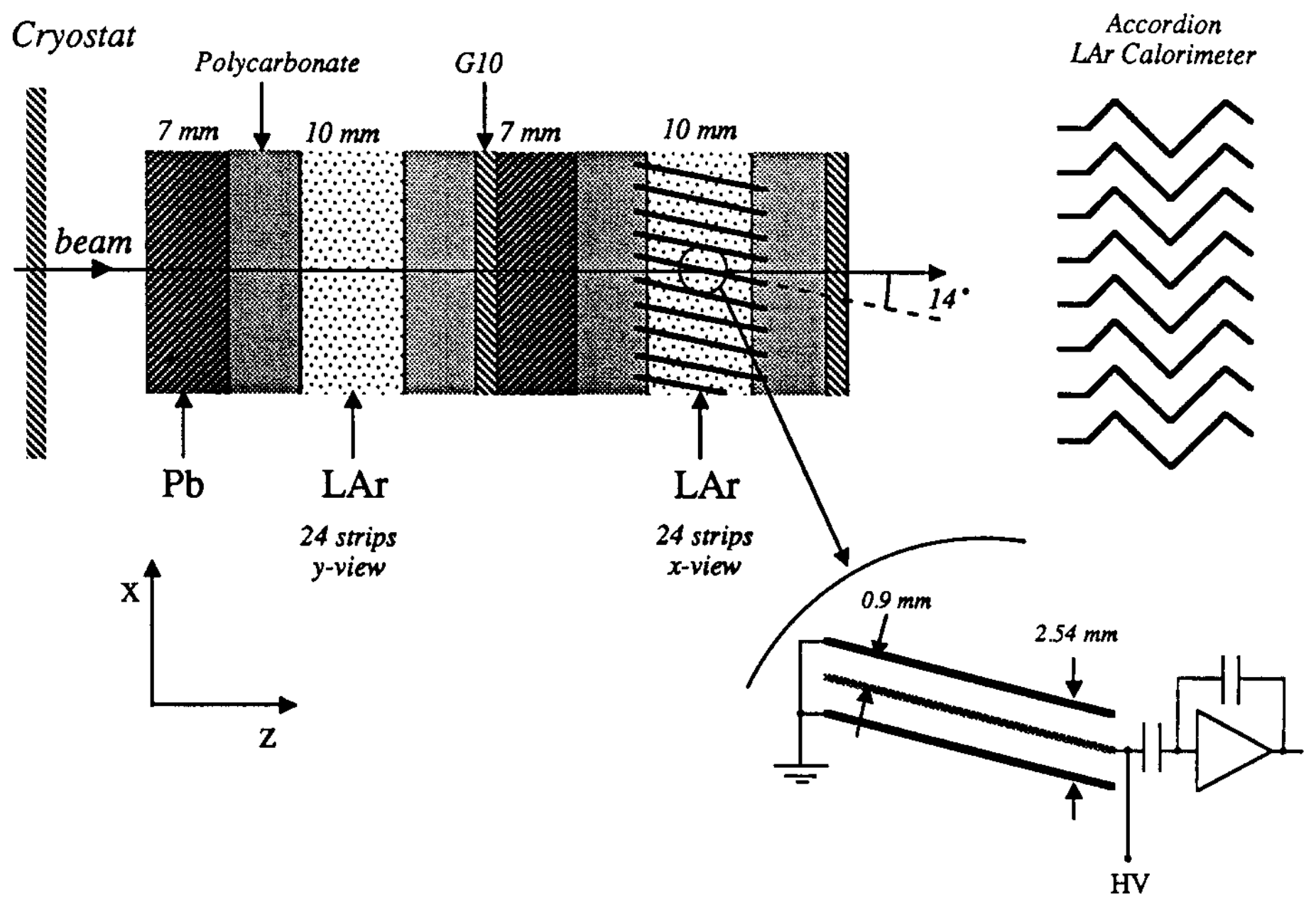

Figure 1: Schematic layout of the LAr preshower prototype (top view, not to scale) and of the geometry of one cell. 


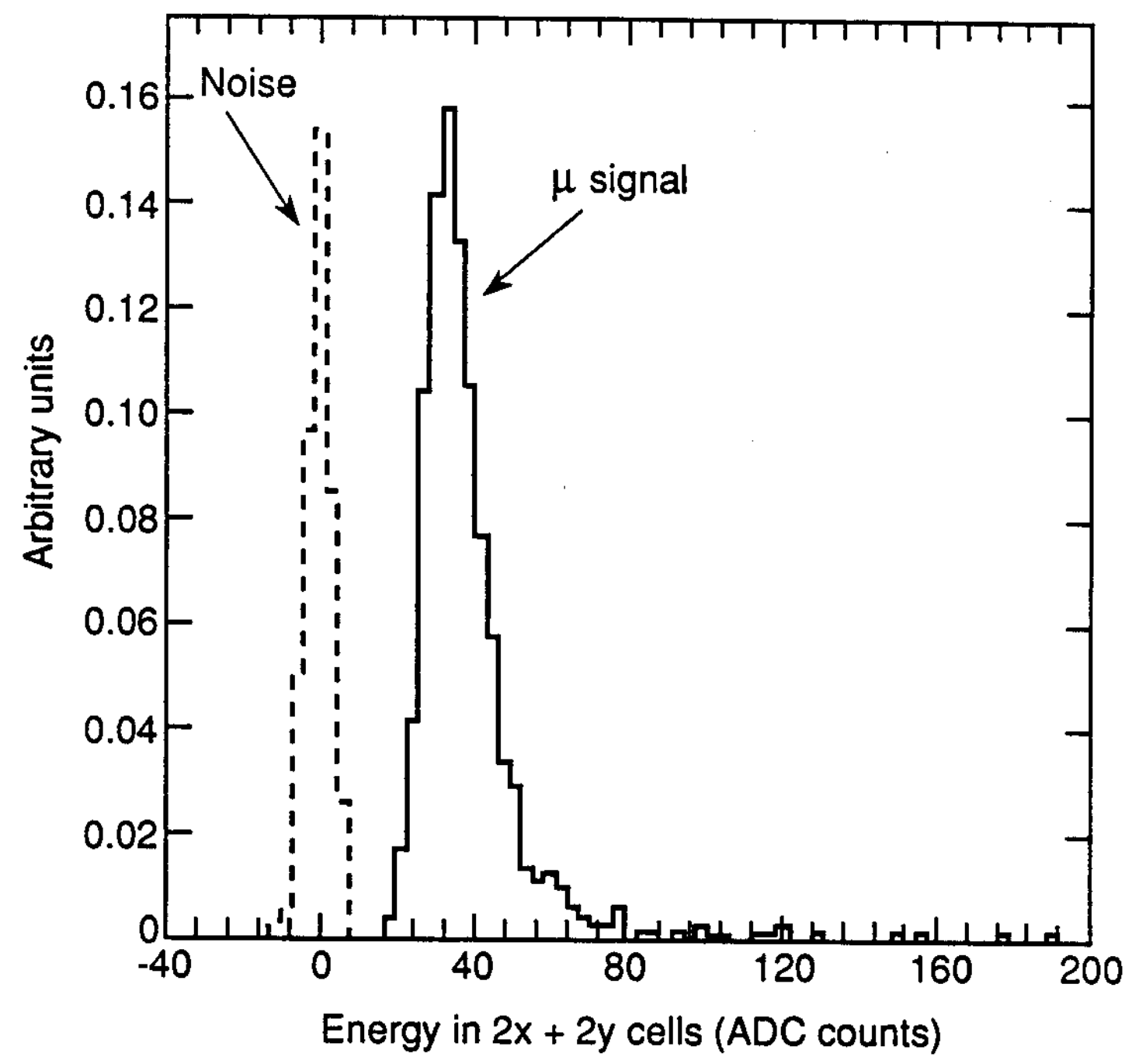

Figure 2: Energy deposited in the two layers of the preshower prototype by $180 \mathrm{GeV}$ muons. The dashed line is the distribution of the electronic noise (see text). 


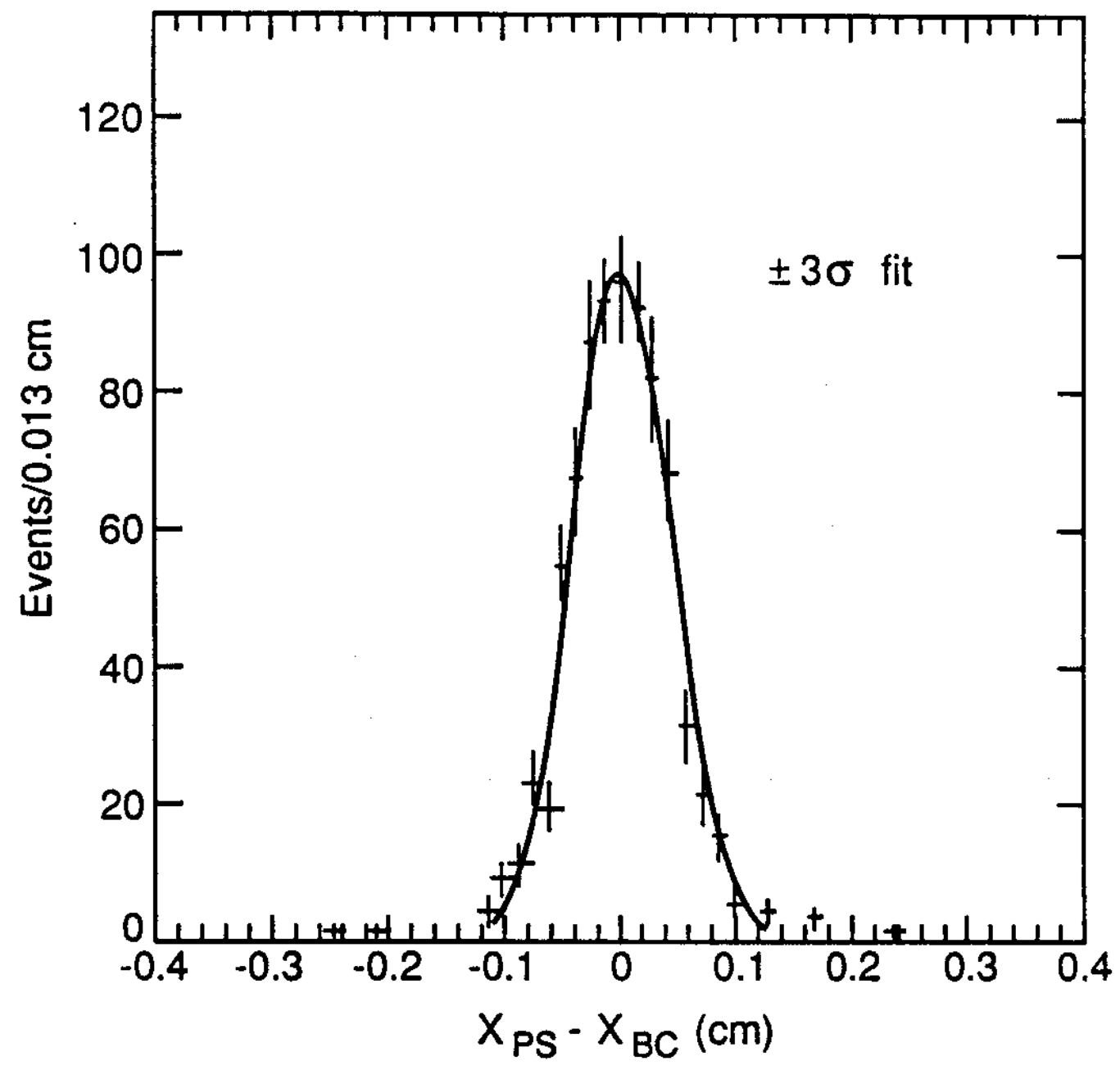

Figure 3: Difference between the muon position reconstructed in the preshower $x$ view and the impact point provided by the beam chambers. The Gaussian fit is superimposed. 

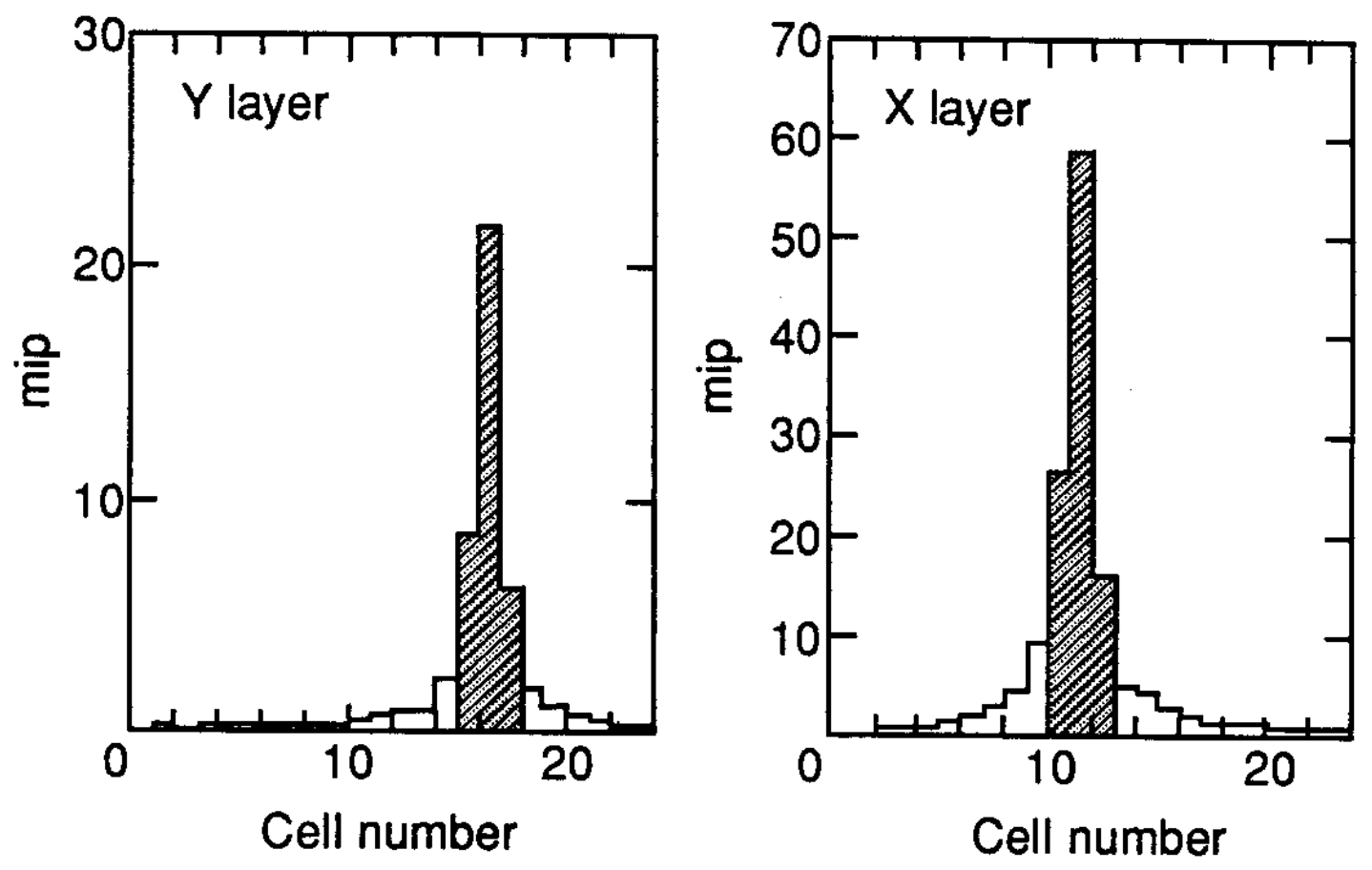

Figure 4: Average shower profile in the two preshower layers for $90 \mathrm{GeV}$ electrons. The dashed region indicates the 3 cell cluster used for the measurement of the shower position. 


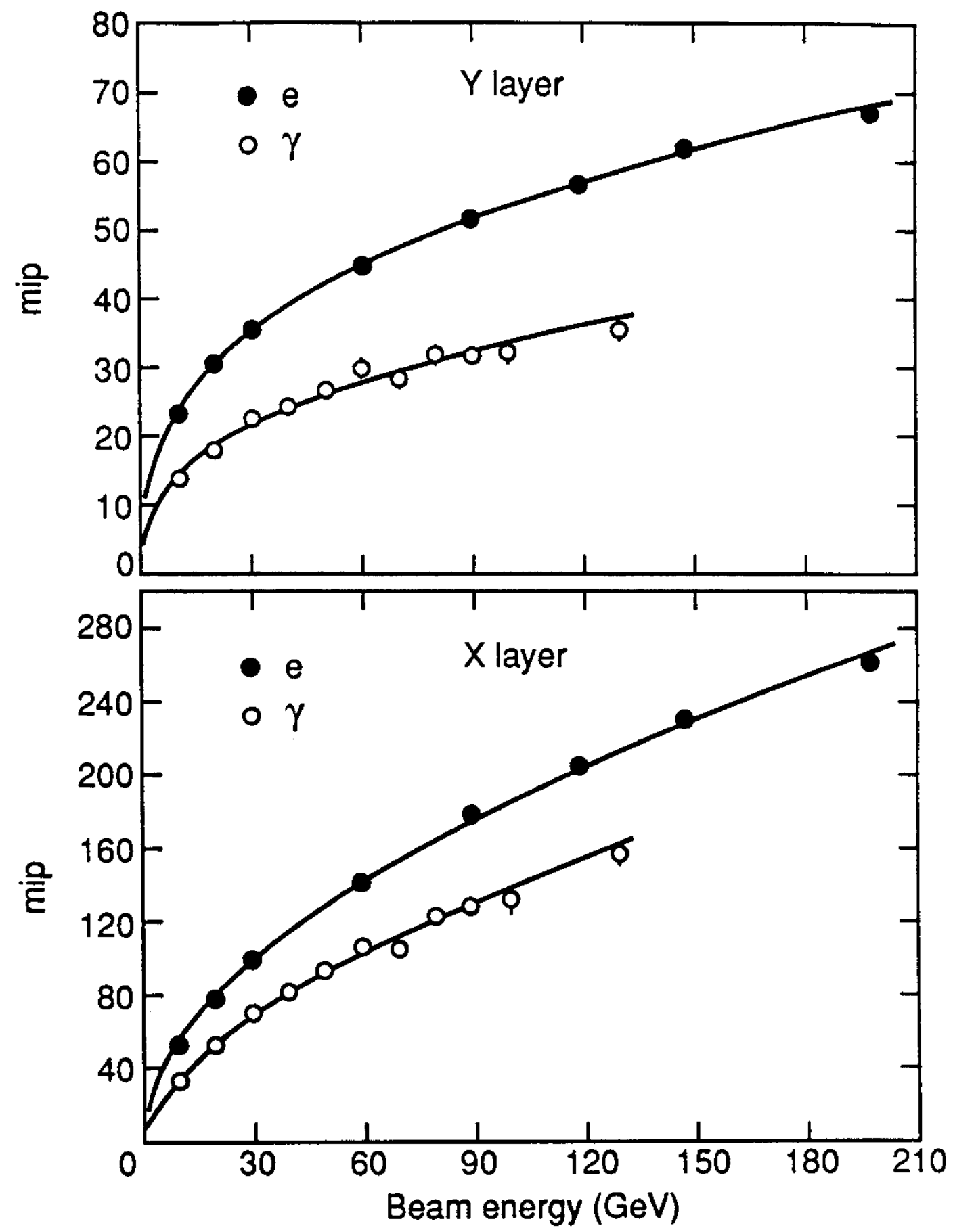

Figure 5: Average signals produced in the two preshower layers by electrons (black points) and photons (open circles, see section 6 ) as a function of the incident beam energy. 


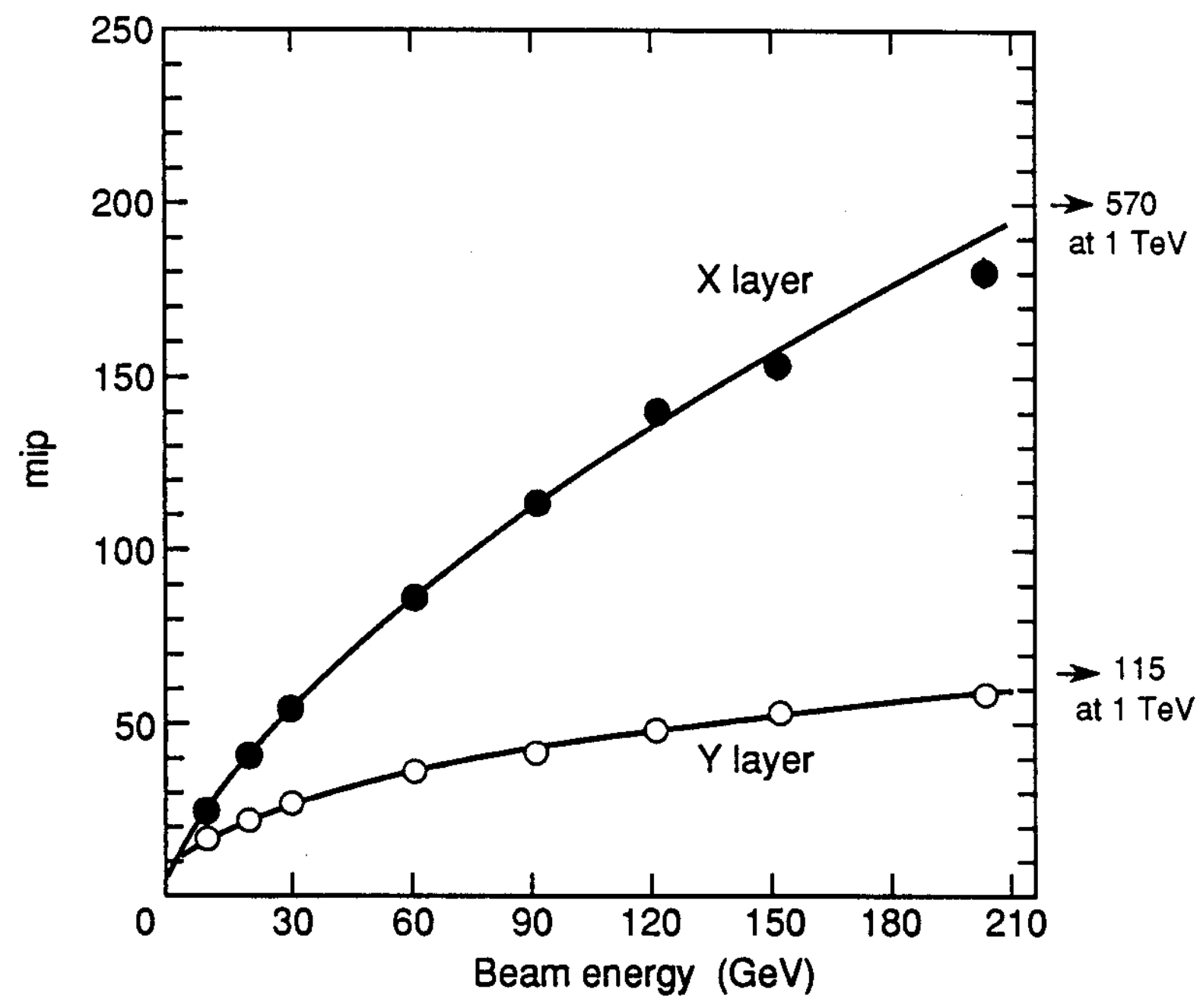

Figure 6: The signal at $95 \%$ efficiency in the most energetic channel of the first (open circles) and second (black points) preshower layer as a function of the electron beam energy. Superimposed two fits of the form $\alpha \cdot E_{b e a m}^{\beta}$. 


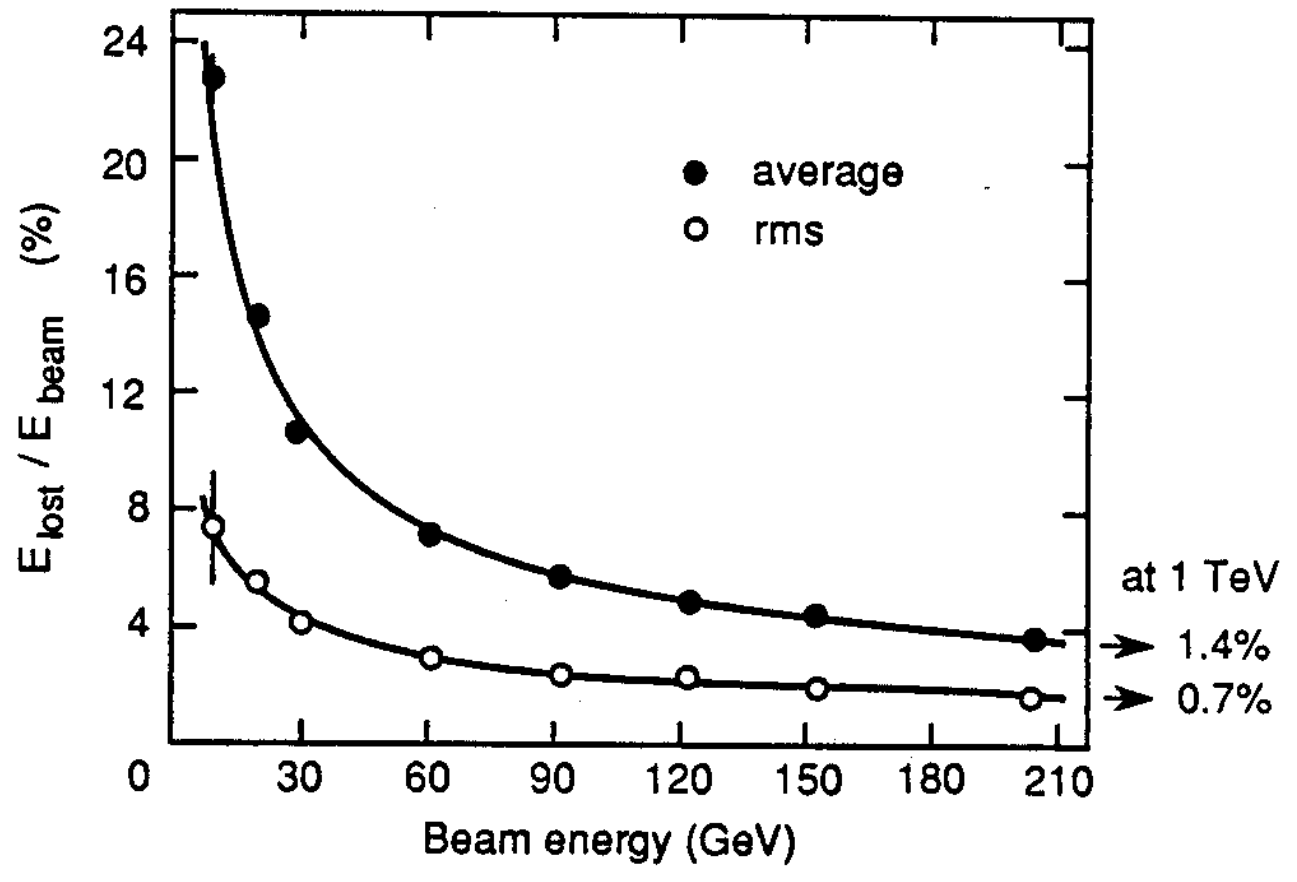

Figure 7: Average (black points) and rms (open circles) fraction of energy lost in the material upstream of the calorimeter versus the incident electron energy. Superimposed the best fits to the experimental points. 


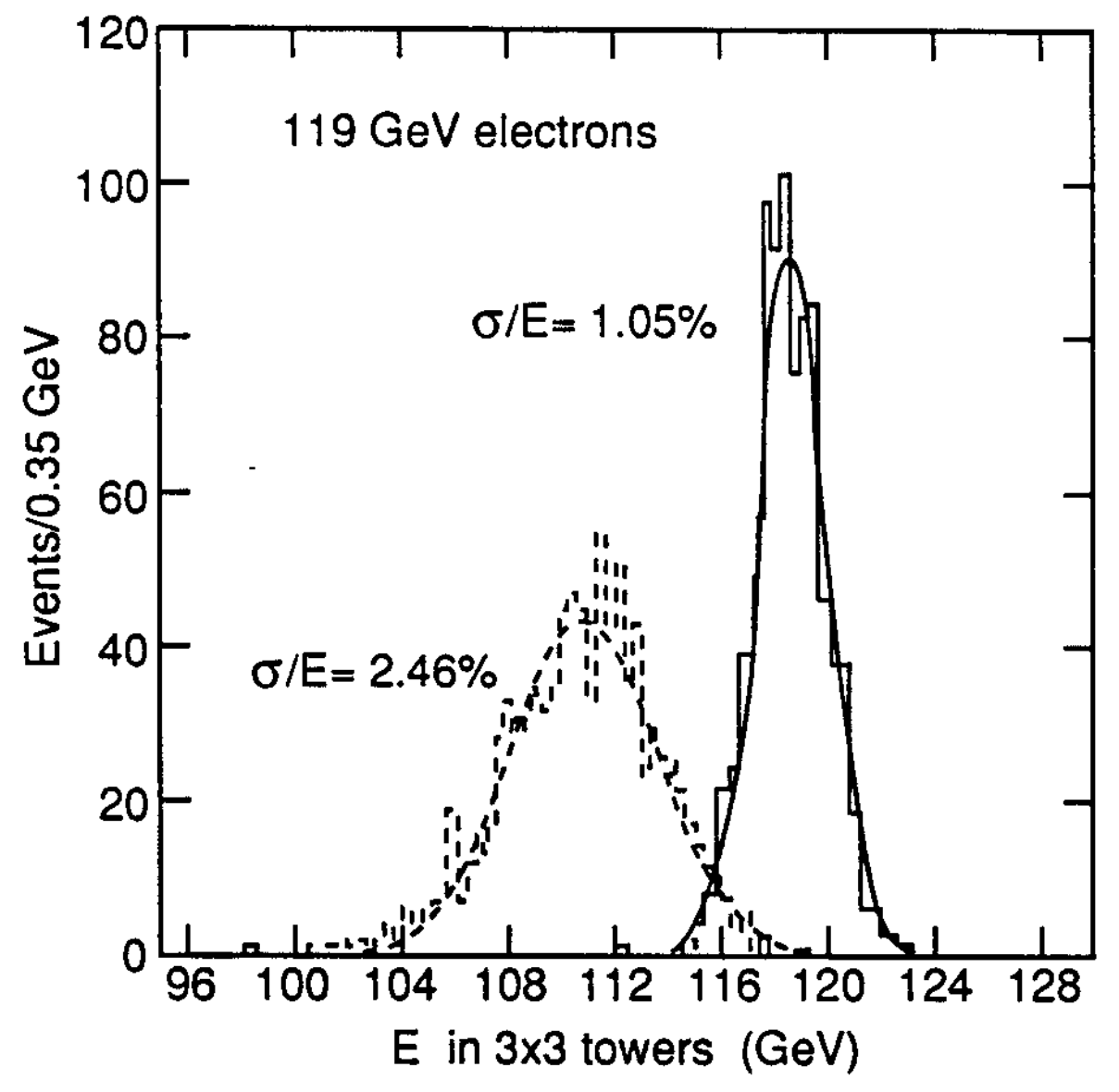

Figure 8: Reconstructed spectrum for $119 \mathrm{GeV}$ electrons before (dashed line) and after (full line) adding the preshower signal to the calorimeter energy. 


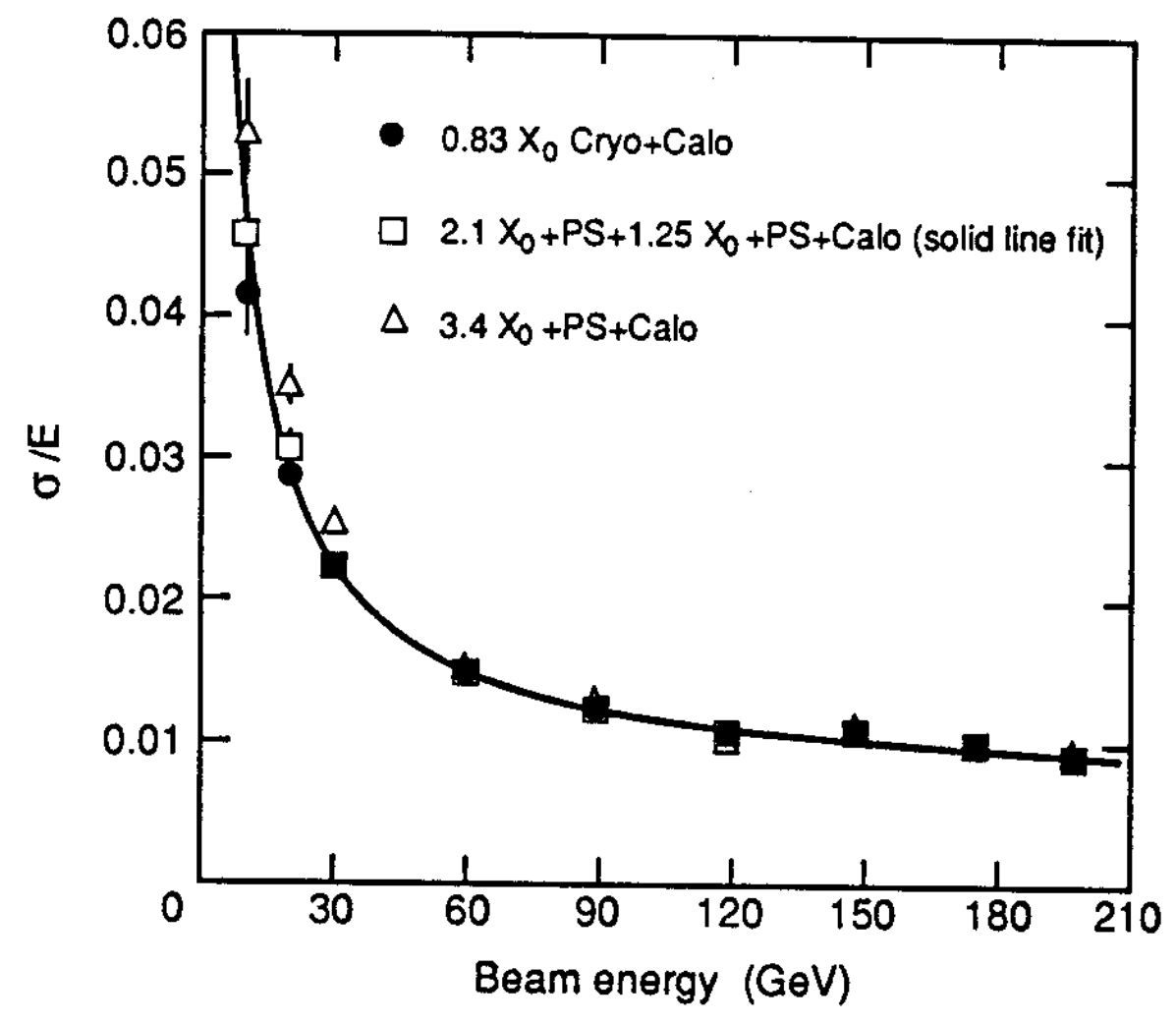

Figure 9: Fractional energy resolution of the Accordion calorimeter versus the incident electron energy in the region covered by the preshower when both (open squares) or only the second (triangles) preshower layer are used for the energy reconstruction. Black points: resolution obtained in the region not covered by the preshower. 


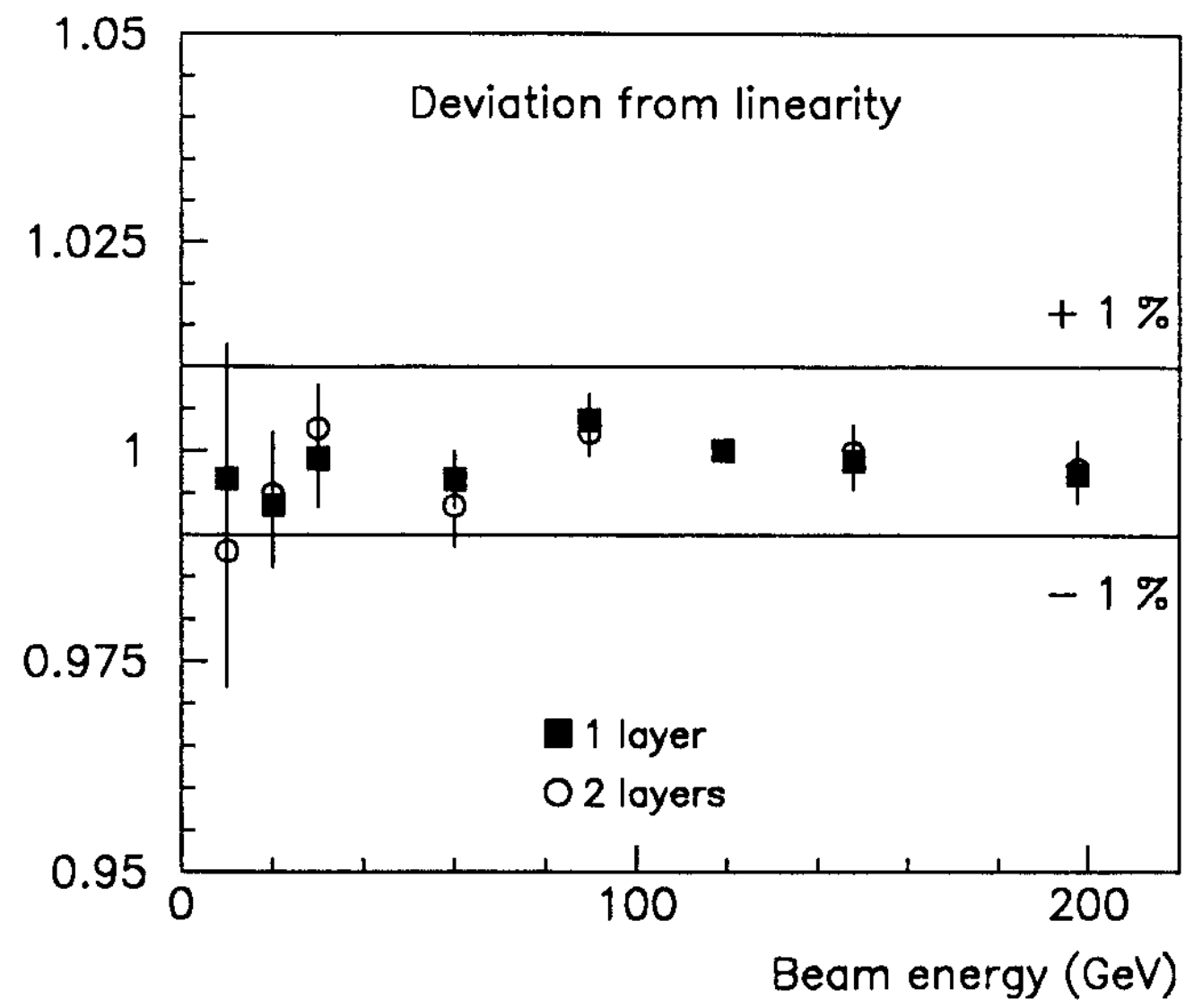

Figure 10: Normalized preshower-calorimeter response as a function of the incident beam energy (fixed to 1 at $119 \mathrm{GeV}$ ) when only the second (black squares) or both (open circles) preshower layers are used for the energy measurement. 


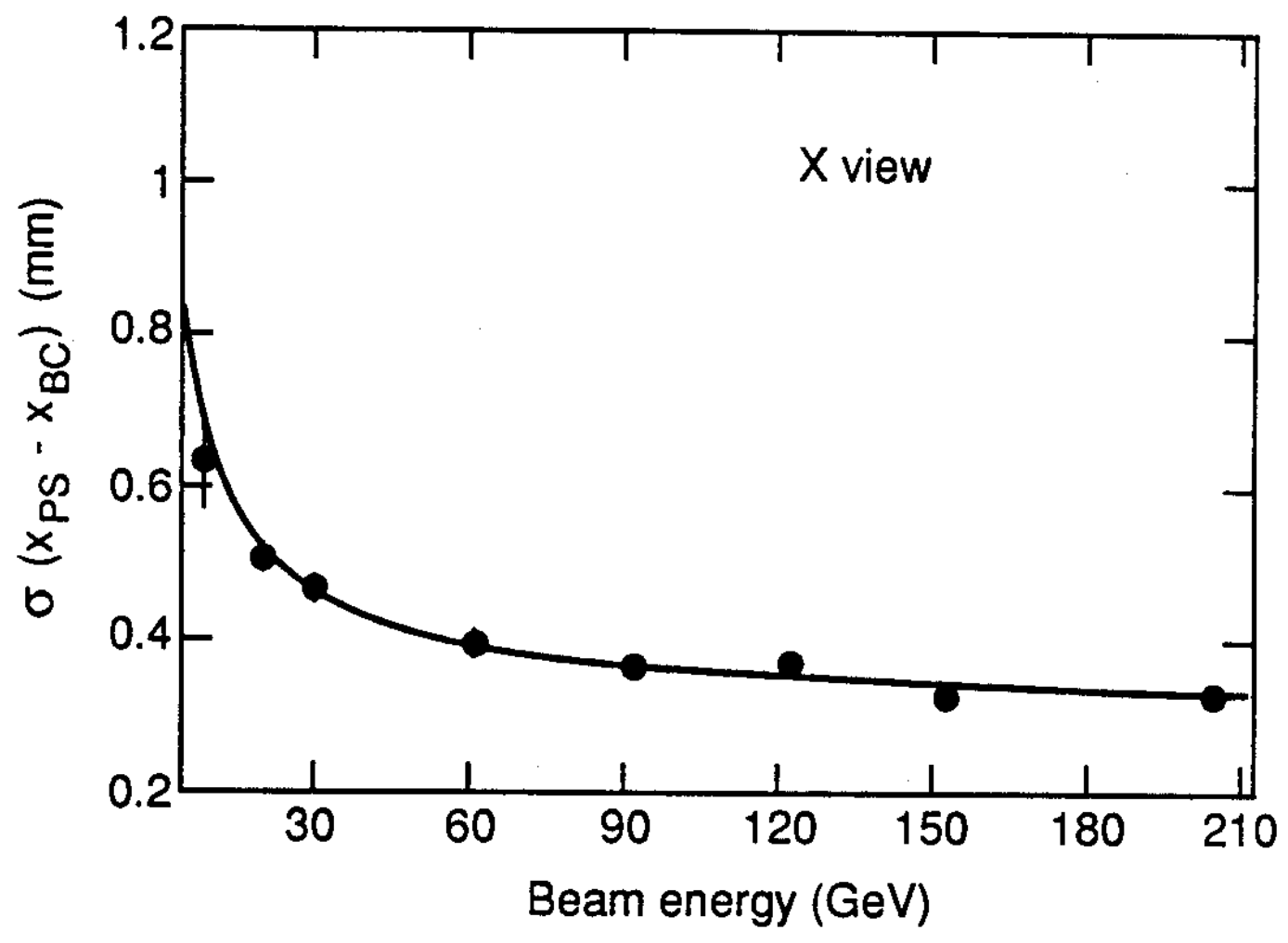

Figure 11: Space resolution of the preshower prototype in the $x$ direction (BC contribution not unfolded) as a function of the incident electron energy. The best fit is superimposed (see text). 


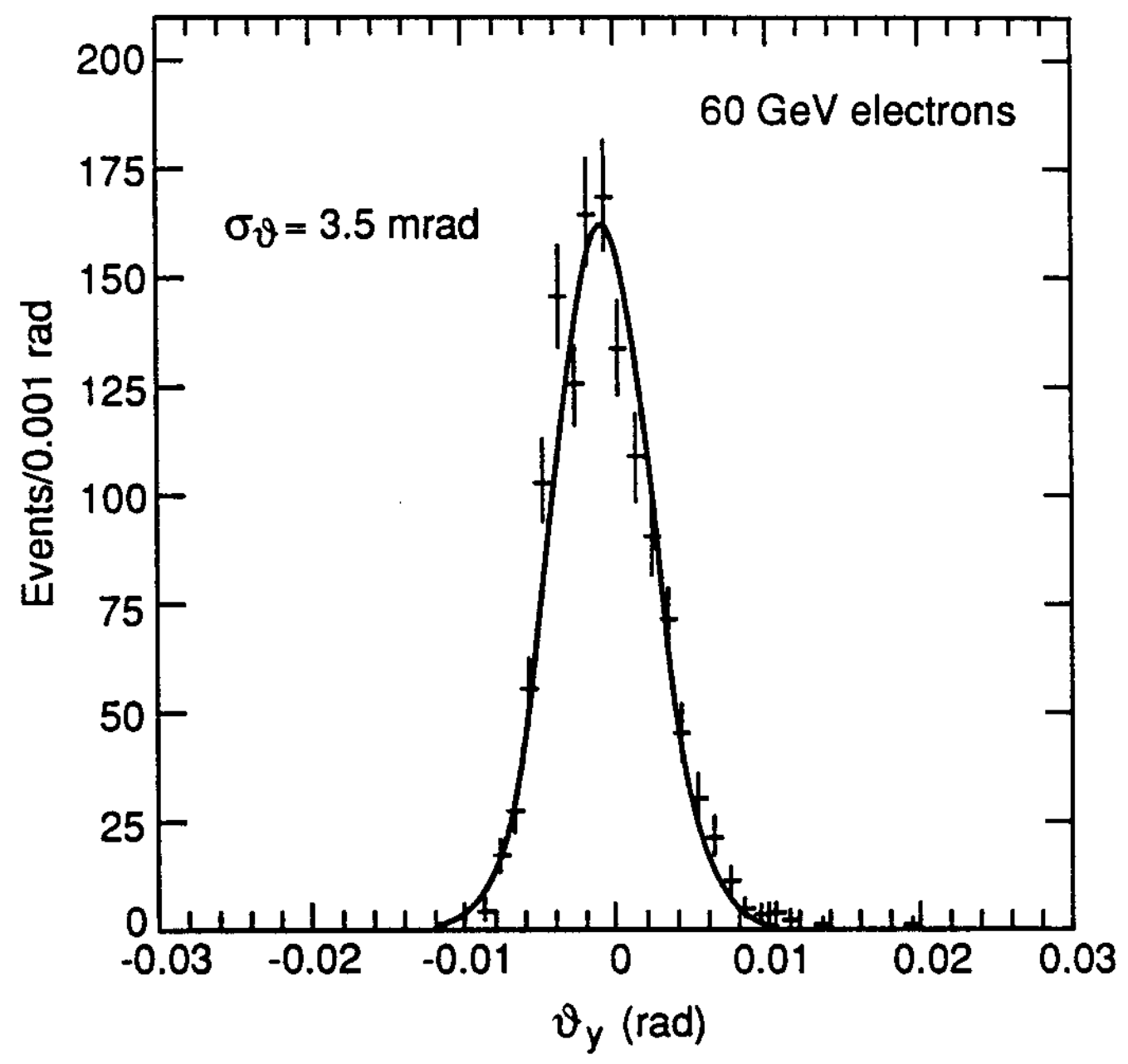

Figure 12: Angle of incidence in the $y$ view measured by the preshower-calorimeter system for $60 \mathrm{GeV}$ electrons perpendicular to the two prototypes. Superimposed is the Gaussian fit. 

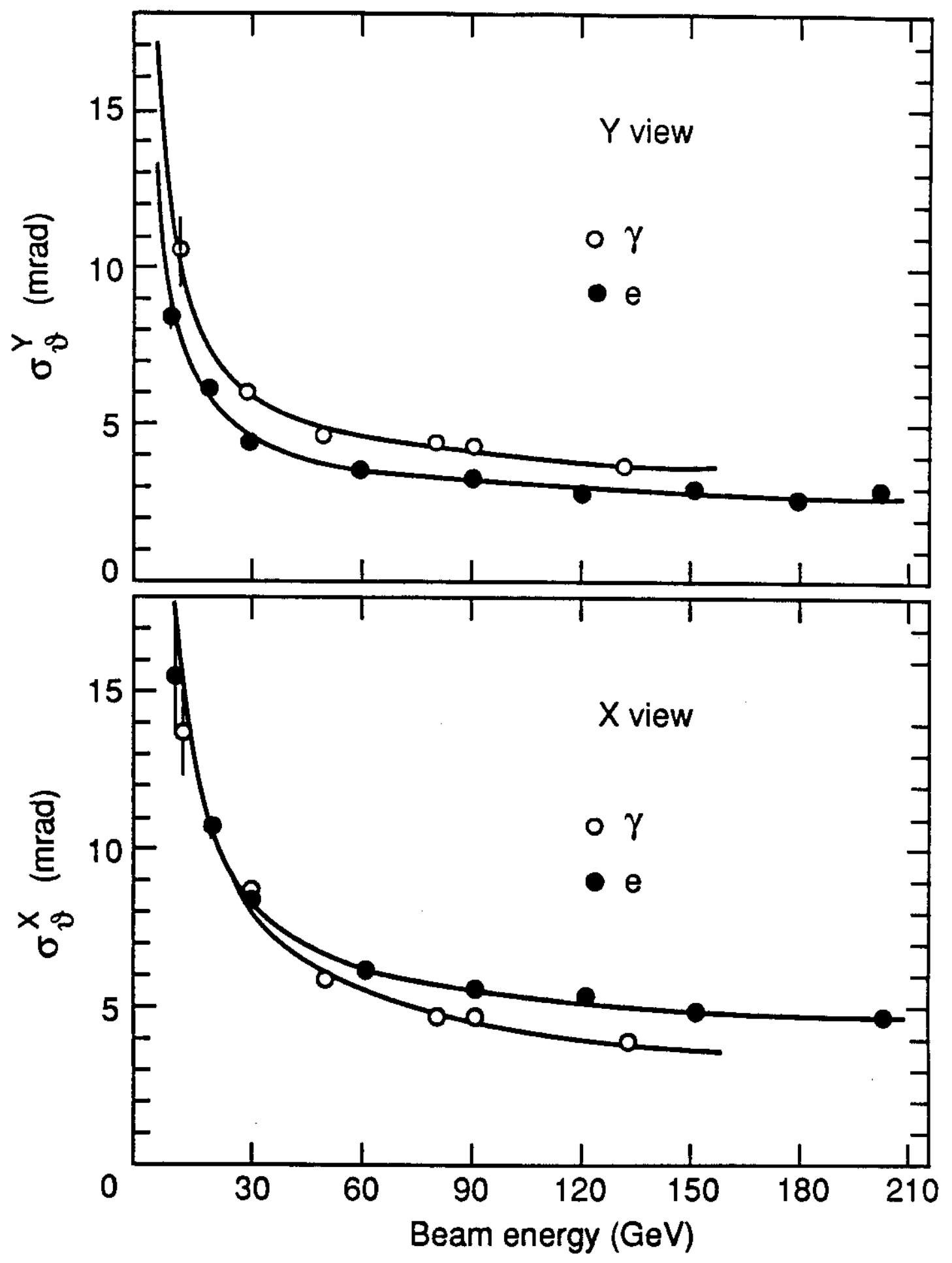

Figure 13: Angular resolution, in the two directions, of the combined preshower-calorimeter system as a function of energy. Black points: electrons. Open circles: photons (see section 6.1). 


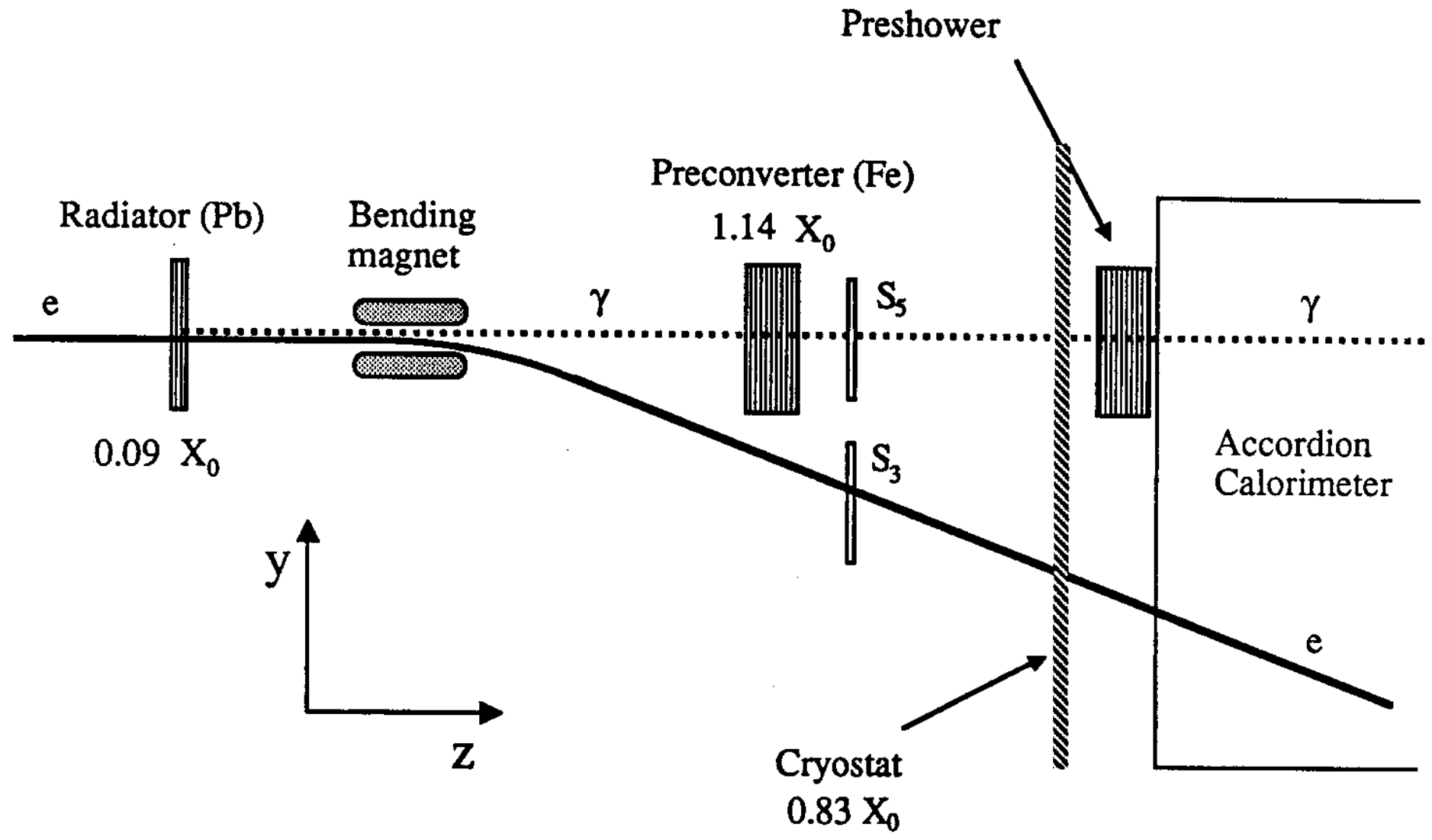

Figure 14: Sketch (not on scale) of the test beam setup used for data taking with photons. 

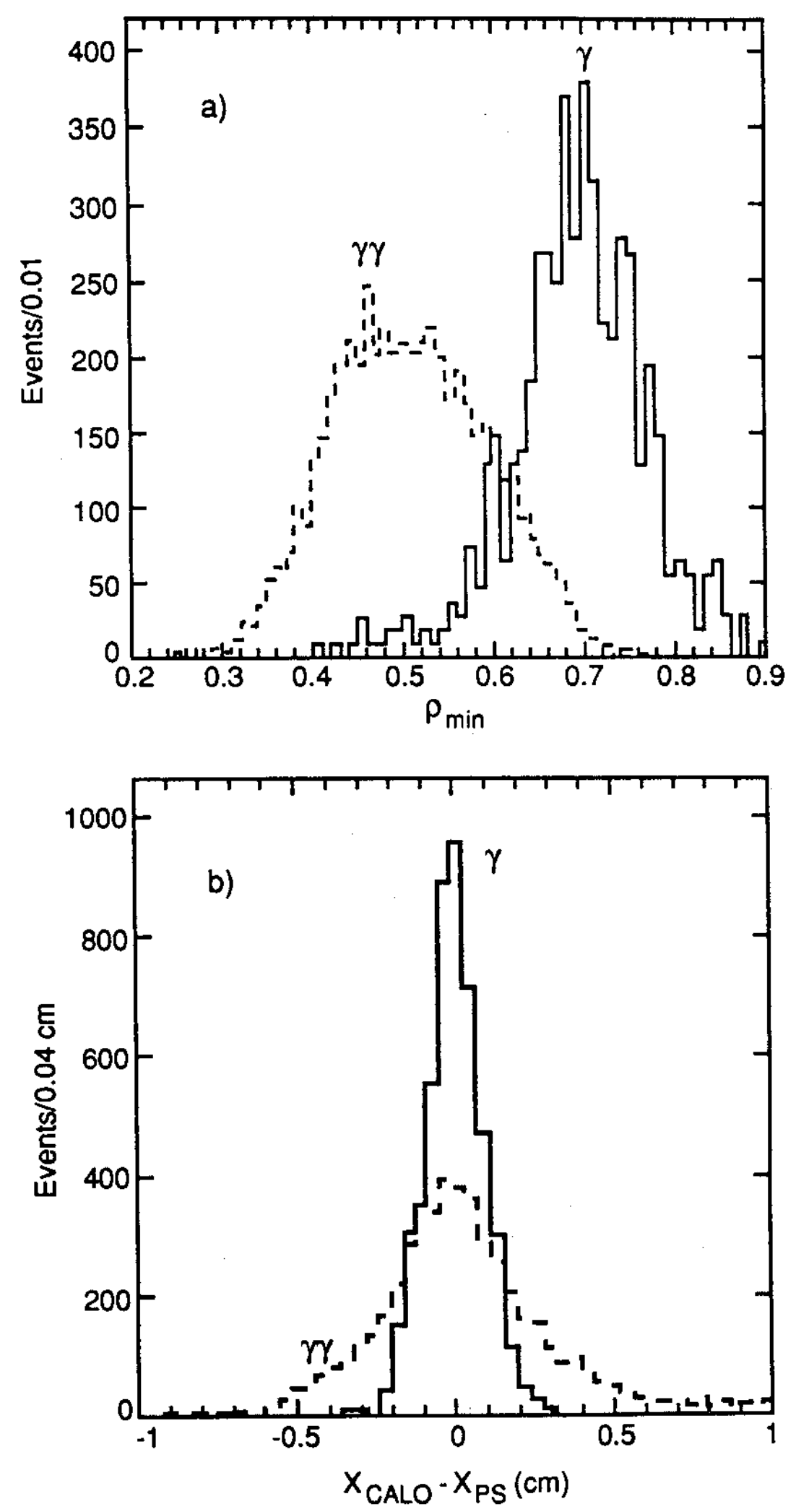

Figure 15: a) Distribution of the shape variable $\rho$ (for each event the lowest value from the two preshower layers is plotted); b) difference between the shower barycentres in the $x$ view measured in the calorimeter and in the preshower. Full line: single $50 \mathrm{GeV}$ photons.

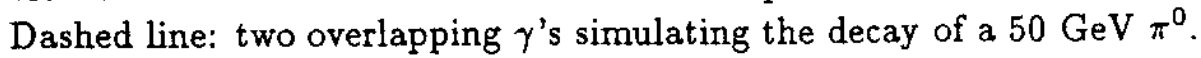




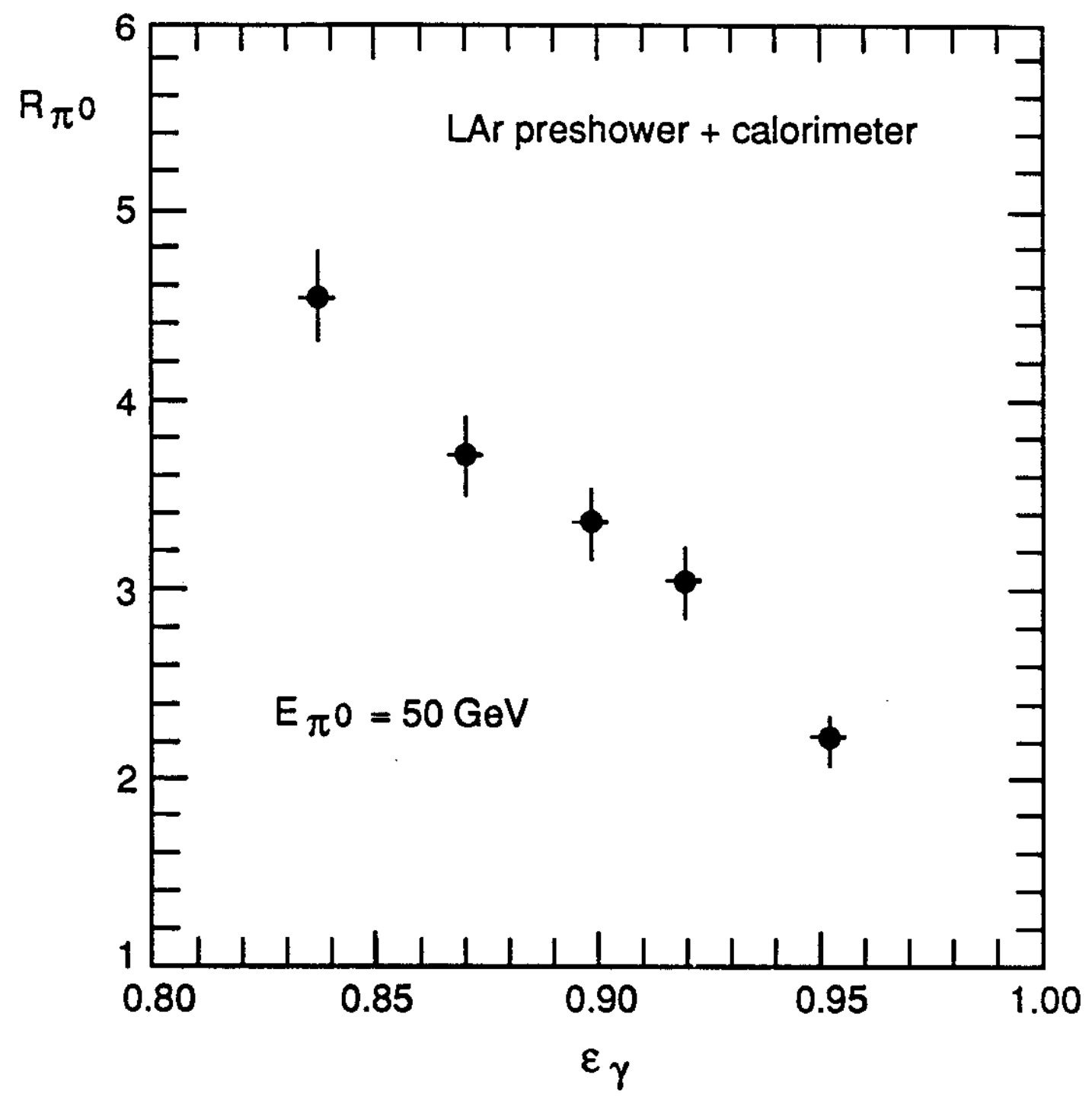

Figure 16: Rejection against $50 \mathrm{GeV} \pi^{0}$ 's provided by the combined preshower-calorimeter system versus the efficiency on single photons. 